Jian Wu, Hongying Xia*, Libo Zhang, Yi Xia, Jinhui Peng, Shixing Wang, Zhaoqiang Zheng and Shengzhou Zhang

\title{
Effect of Microwave Heating Conditions on the Preparation of High Surface Area Activated Carbon from Waste Bamboo
}

\begin{abstract}
The present study reports the effect of microwave power and microwave heating time on activated carbon adsorption ability. The waste bamboo was used to preparing high surface area activated carbon via microwave heating. The bamboo was carbonized for $2 \mathrm{~h}$ at $600^{\circ} \mathrm{C}$ to be used as the raw material. According to the results, microwave power and microwave heating time had a significant impact on the activating effect. The optimal $\mathrm{KOH} / \mathrm{C}$ ratio of 4 was identified when microwave power and microwave heating time were $700 \mathrm{~W}$ and 15 min, respectively. Under the optimal conditions, surface area was estimated to be $3441 \mathrm{~m}^{2} / \mathrm{g}$ with pore volume of $2.093 \mathrm{ml} / \mathrm{g}$ and the significant proportion of activated
\end{abstract}

carbon was microporous (62.3\%). The results of Fourier transform infrared spectroscopy (FTIR) were illustrated that activated carbon surface had abundant functional groups. Additionally the pore structure is characterized using Scanning Electron Microscope (SEM).

Keywords: high surface area activated carbon, microwave heating, waste bamboo

PACS $^{\circledR}$ (2010). 81.05.Rm

DOI 10.1515/htmp-2014-0096

Received June 6, 2014; accepted October 9, 2014

\footnotetext{
*Corresponding author: Hongying Xia, Yunnan Provincial Key Laboratory of Intensification Metallurgy, Kunming, Yunnan 650093, China; National Local Joint Laboratory of Engineering Application of Microwave Energy and Equipment Technology, Kunming, Yunnan 650093, China; Key Laboratory of Unconventional Metallurgy, Ministry of Education, Kunming, Yunnan 650093, China; Faculty of Metallurgical and Energy Engineering, Kunming University of Science and Technology, Kunming 650093, China, E-mail: hyxia81@hotmail.com Jian Wu, Yunnan Provincial Key Laboratory of Intensification Metallurgy, Kunming, Yunnan 650093, China; National Local Joint Laboratory of Engineering Application of Microwave Energy and Equipment Technology, Kunming, Yunnan 650093, China; Key Laboratory of Unconventional Metallurgy, Ministry of Education, Kunming, Yunnan 650093, China; Faculty of Metallurgical and Energy Engineering, Kunming University of Science and Technology, Kunming 650093, China, E-mail: xiaoyingyue123@163.com Libo Zhang, Yunnan Provincial Key Laboratory of Intensification Metallurgy, Kunming, Yunnan 650093, China; National Local Joint Laboratory of Engineering Application of Microwave Energy and Equipment Technology, Kunming, Yunnan 650093, China; Key Laboratory of Unconventional Metallurgy, Ministry of Education, Kunming, Yunnan 650093, China; Faculty of Metallurgical and Energy Engineering, Kunming University of Science and Technology, Kunming 650093, China, E-mail: libozh77@yahoo.com Yi Xia, Yunnan Provincial Key Laboratory of Intensification Metallurgy, Kunming, Yunnan 650093, China; National Local Joint Laboratory of Engineering Application of Microwave Energy and Equipment Technology, Kunming, Yunnan 650093, China; Key Laboratory of Unconventional Metallurgy, Ministry of Education, Kunming, Yunnan 650093, China, E-mail: 58351260@qq.com

Jinhui Peng, Yunnan Provincial Key Laboratory of Intensification Metallurgy, Kunming, Yunnan 650093, China; National Local Joint Laboratory of Engineering Application of Microwave Energy and Equipment Technology, Kunming, Yunnan 650093, China; Key Laboratory of Unconventional Metallurgy, Ministry of Education, Kunming, Yunnan 650093, China; Faculty of Metallurgical and Energy Engineering, Kunming University of Science and Technology, Kunming 650093, China, E-mail: jhpeng@kmust.edu.cn

Shixing Wang, Yunnan Provincial Key Laboratory of Intensification Metallurgy, Kunming, Yunnan 650093, China; National Local Joint Laboratory of Engineering Application of Microwave Energy and Equipment Technology, Kunming, Yunnan 650093, China; Key Laboratory of Unconventional Metallurgy, Ministry of Education, Kunming, Yunnan 650093, China; Faculty of Metallurgical and Energy Engineering, Kunming University of Science and Technology, Kunming 650093, China, E-mail: 175379478@qq.com

Zhaoqiang Zheng, Yunnan Provincial Key Laboratory of Intensification Metallurgy, Kunming, Yunnan 650093, China; National Local Joint Laboratory of Engineering Application of Microwave Energy and Equipment Technology, Kunming, Yunnan 650093, China; Key Laboratory of Unconventional Metallurgy, Ministry of Education, Kunming, Yunnan 650093, China; Faculty of Metallurgical and Energy Engineering, Kunming University of Science and Technology, Kunming 650093, China, E-mail: zhijianyuan2008@126.com

Shengzhou Zhang, Yunnan Provincial Key Laboratory of Intensification Metallurgy, Kunming, Yunnan 650093, China; National Local Joint Laboratory of Engineering Application of Microwave Energy and Equipment Technology, Kunming, Yunnan 650093, China; Key Laboratory of Unconventional Metallurgy, Ministry of Education, Kunming, Yunnan 650093, China; Faculty of Metallurgical and Energy Engineering, Kunming University of Science and Technology, Kunming 650093, China, E-mail: 249928712@qq.com
} 


\section{Introduction}

Activated carbon is a material that has abundant pore structure and high adsorption ability. Activated carbon which is widely used in many fields such as environmental, chemical, metallurgy, food processing and pharmaceutical has become an indispensable product in national economic development [1]. Activated carbon is produced from different raw carbon resources like wood, sawdust, coconut shell, almond shell and feces of silkworms [2-5]. Bamboo is a green construction material that has a lot of advantages such as capability of being reused or recycled, sustainability and environmental friendliness. Bamboo has been used in lots of fields such as construction, clothing, household appliances and entertainment materials [6-8]. However, waste bamboo materials are often dumped or burnt as wastes, which causes some serious pollution problems. Activated carbon which is prepared by waste bamboo has a broad prospect in our society. Moreover, bamboobased activated carbon has been used for dyes, ammonia and so on $[9,10]$.

The activation process of activated carbon is mostly divided into two categories: physical activation process and chemical activation process [11, 12]. In physical activation process, carbon dioxide [13] and steam [14] are often used to make carbonized material mild oxidation. In chemical activation process, there are some activating agents widely used such as $\mathrm{KOH}$ [15-19], $\mathrm{ZnCl}_{2}$ [20], $\mathrm{K}_{2} \mathrm{CO}_{3}[21,22]$ and $\mathrm{H}_{3} \mathrm{PO}_{4}$ [23].

A conventional heating method is widely adopted in the preparation of activated carbon; however, it has some disadvantages such as long heating time and high energy consumption [24, 25]. Microwave heating is a new technique for the production of materials and it is widely used in the preparation and regeneration of activated carbon. Microwave heating has been proved beyond doubt to possess qualities such as fast heating, energy efficient, easy to control, small thermal inertia and selective heating [26-30].

The present work attempts to utilize microwave heating to prepare activated carbon from waste bamboo. The effects of microwave power and microwave heating time to activated carbon using $\mathrm{KOH}$ to be the activating agent have been studied. The resultant products are characterized by using the pore structure, pore size distribution, Fourier transform infrared spectroscopy (FTIR) analysis and Scanning Electron Microscope (SEM) analysis.

\section{Experimental}

\section{Materials}

Waste bamboo is selected as the raw material for activated carbon. The main reagents utilized in the experiments are $\mathrm{KOH}(\mathrm{AR})$ and hydrochloric acid (AR).

\section{Methods}

Waste bamboo removed of impurities was put into the crucible and heated to a carbonization temperature of $600^{\circ} \mathrm{C}$ at a heating rate of $10^{\circ} \mathrm{C} / \mathrm{min}$ and held for $2 \mathrm{~h}$ in an inert atmosphere. The carbonized material is put out from muffle furnace and crushed more than 600-1,000 $\mu \mathrm{m}$ after it is cooling to room temperature in the muffle furnace. The carbonized material was put into the bottle after drying enough. The carbonized material was mixed with $\mathrm{KOH} / \mathrm{C}$ mass ratio of 4 uniformly and put into the microwave furnace with crucible with heating at $\mathrm{N}_{2}$ atmosphere. High-temperature material after microwave heating was transferred to distilled water for the formation of pores. The activated carbon was washed with 1:1 volume of hydrochloric acid $(0.1 \mathrm{~mol} / \mathrm{l})$, followed by rinsing with distilled water with $\mathrm{pH}$ value more than 6-7. Finally, samples were filtered, dried at $110^{\circ} \mathrm{C}$ for $4 \mathrm{~h}$ to be high surface area activated carbon.

Microwave heating apparatus was developed by Yunnan Provincial Key Laboratory of Intensification Metallurgy. The microwave frequency was 2,450 MHz. The maximum microwave output power was $700 \mathrm{~W}$ and power was continuously adjustable.

\section{Characterization of activated carbon}

The nitrogen adsorption isotherm of activated carbon is measured by automatic physical and chemical adsorption instrument (Autosorb-1-C, Quantachrome) at 77 K. Specific surface area of activated carbon is calculated by BET method and total pore volume is determined by nitrogen adsorption isotherm until a relative pressure of $p / p_{0}$ of 0.98. T-plot method is used to calculate micropore volume and external specific surface area. The pore size distribution is determined by the nitrogen adsorption isotherm generated utilizing non-localization density functional theory (NLDFT). FTIR spectra are generated in the range of $4,000-400 \mathrm{~cm}^{-1}$ by using Nicolet 8700 spectrophotometer. The transmission spectra of samples are prepared by mixing with $\mathrm{KBr}$ crystals and pressed into a pellet. 


\section{Results and discussion}

\section{Effect of microwave power}

\section{Pore structure analysis}

Effect of microwave power to activated carbon adsorption ability, yield and pore size distribution has been studied at optimal conditions. The nitrogen adsorption isotherms of the activated carbon prepared with different activating agents are shown in Figure 1. The condition of microwave power experiment is microwave heating time of $15 \mathrm{~min}$. The pore structural parameters deduced from the isotherms are summarized in Table 1. Pore size distribution of activated carbon characterized through NLDFT is shown in Figure 2.

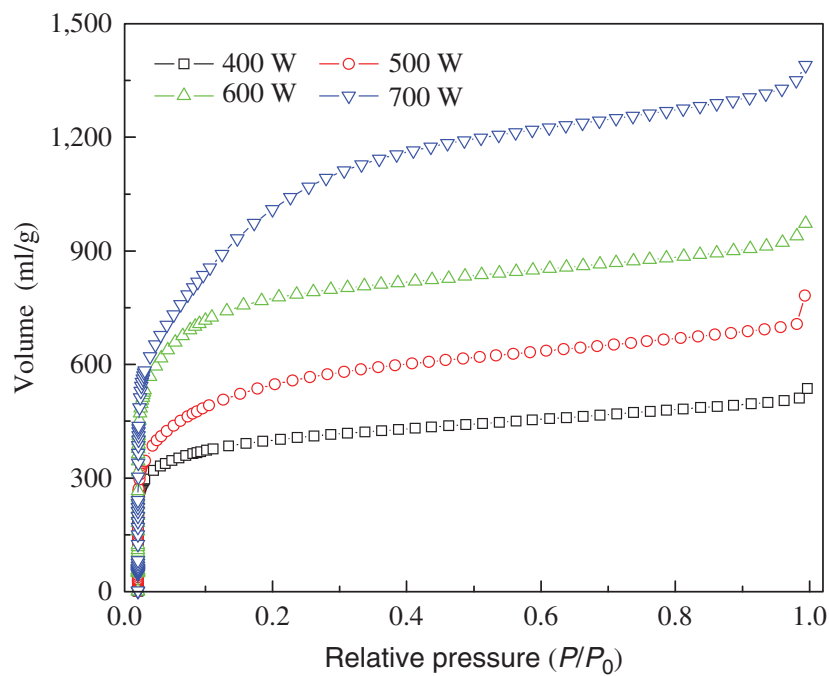

Figure 1: Nitrogen adsorption isotherm of the activated carbon with different microwave power.

As can be seen from Figure 1, the isotherms based on the International Union of Pure Applied Chemistry (IUPAC) [31] classification can be categorized to be type I isotherm. It is illustrated that main pore of activated carbon is micropore and nitrogen adsorption increases with relative pressure increase. Adsorption

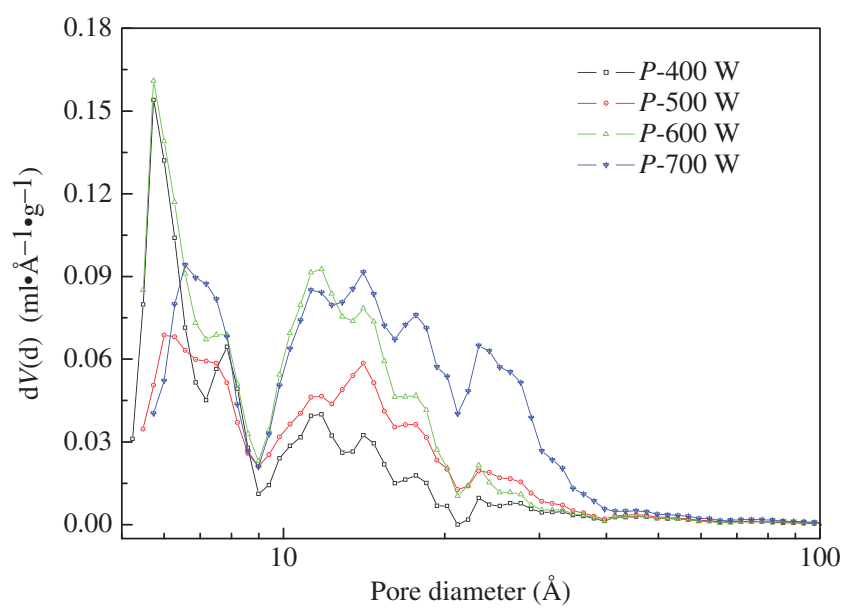

Figure 2: Pore size distribution chart for different microwave power.

ability reaches more than $60 \%$ of saturated adsorption ability at a $p / p_{0}$ of 0.1 . Adsorption ability increases with relative pressure increases beyond a $p / p_{0}$ of 0.1 ; however, there is a slow increasing trend of the isotherm and the adsorption isotherm is almost horizontal. Adsorption ability increases shapely and has tailing phenomenon until a $p / p_{0}$ of 1 , which indicates that activated carbon contains a certain amount of mesopores and macropores.

Table 1 shows pore structure parameters such as BET surface area and total pore volume. With microwave power increasing, BET surface area, total pore volume, micropore surface area, external surface area, micropore volume and mesopore volume increase. At the same time, average pore size decreases with power increase. The micropore contents of activated carbon exceeded $60 \%$ at different power, which means activated carbon is micropore style.

Pore size distribution of activated carbon with the different microwave power is presented in Figure 2. According to IUPAC classification, pore size distribution of activated carbon is concentrated in the micropore field. With microwave power increasing, pore volume has a significant increase. Pore size distribution is widely spread.

Table 1: Details of pore structure of activated carbon with different microwave power.

\begin{tabular}{lrrrrrrrr}
\hline Microwave power $(\mathrm{W})$ & $\boldsymbol{S}_{\text {BET }}\left(\mathrm{m}^{2} / \mathrm{g}\right)$ & $V_{\text {tot }}(\mathrm{ml} / \mathrm{g})$ & $\boldsymbol{D}(\mathrm{nm})$ & $V_{\text {mic }}(\mathrm{ml} / \mathrm{g})$ & $\boldsymbol{S}_{\text {mic }}\left(\mathrm{m}^{2} / \mathrm{g}\right)$ & $V_{\text {mes }}(\mathrm{ml} / \mathrm{g})$ & $\boldsymbol{S}_{\text {external }}\left(\mathrm{m}^{2} / \mathrm{g}\right)$ & $V_{\text {mic }} / V_{\text {tot }}(\%)$ \\
\hline 400 & 1,208 & 0.792 & 2.624 & 0.498 & 908 & 0.295 & 300.1 \\
500 & 1,793 & 1.096 & 2.445 & 0.720 & 1,427 & 0.376 & 62.85 \\
600 & 2,347 & 1.430 & 2.438 & 1.006 & 1,914 & 0.424 & 65.3 & 433.1 \\
700 & 3,441 & 2.093 & 2.434 & 1.304 & 2,591 & 0.789 & 850.1 & 62.35 \\
\hline
\end{tabular}




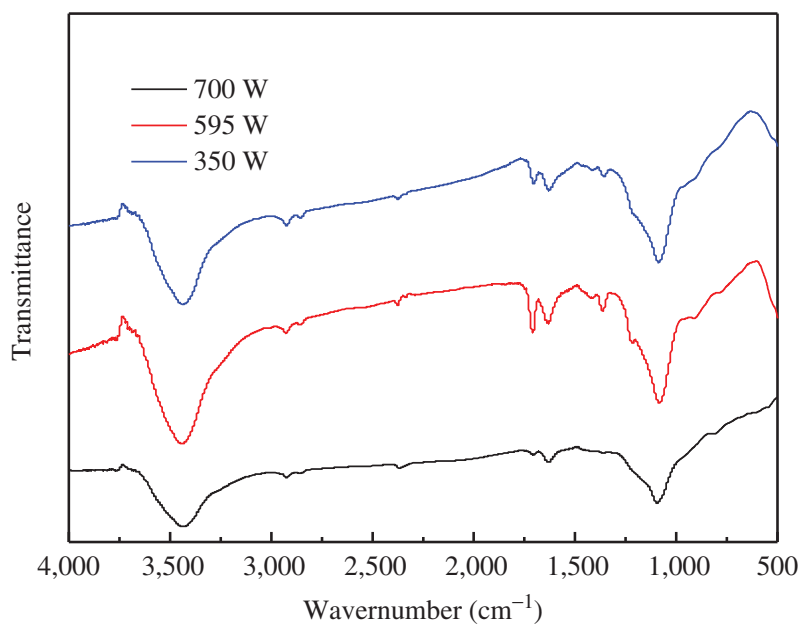

Figure 3: FTIR spectra of activated carbon at different microwave power from waste bamboo.

\section{FTIR analysis}

Figure 3 is the FTIR analysis of the activated carbon at different microwave power. As can be seen from Figure 3, FTIR spectra of activated carbon at different microwave power are similar, with the major peaks observed at $3,435,1,705-1,630,1,085$ and $913-780 \mathrm{~cm}^{-1}$. The band at around $3,435 \mathrm{~cm}^{-1}$ can be assigned to the $-\mathrm{OH}$ stretching vibration mode of hydroxyl functional groups. The band at $1,705 \mathrm{~cm}^{-1}$ indicates that there may exist $C=0$ functional groups and the band at around $1,630 \mathrm{~cm}^{-1}$ can be assigned to $C=\mathrm{C}$ symmetrical stretching of pyrone and $C=0$ of carboxylic groups and the band at $1,090 \mathrm{~cm}^{-1}$ is assigned to be $\mathrm{C}-\mathrm{O}$ stretching vibration mode. The absorption peak at $1,210 \mathrm{~cm}^{-1}$ disappears at $700 \mathrm{~W}$ and the adsorption peak at $913-780 \mathrm{~cm}^{-1}$ is weakened, which obviously means $\mathrm{C}-\mathrm{H}$ outer surface rocking vibration absorption is weak and carbon network structure is large.

\section{Activation yield analysis}

Figure 4 illustrates the effects of microwave power on the activation yield of activated carbon. It can be seen from Figure 4 that activation yield of activated carbon decreases with microwave power increase. With the microwave power increase, the material temperature increases and the reaction rate accelerates. At the same time, the ignition loss degree of activated carbon is larger. This is why activation yield decreases. When microwave power is $700 \mathrm{~W}$, yield is $39.82 \%$. Similar yields have been reported in literatures for other precursors, namely for holm-oak sawdust (25.5\%) and rockrose $(20 \%)$ [32].

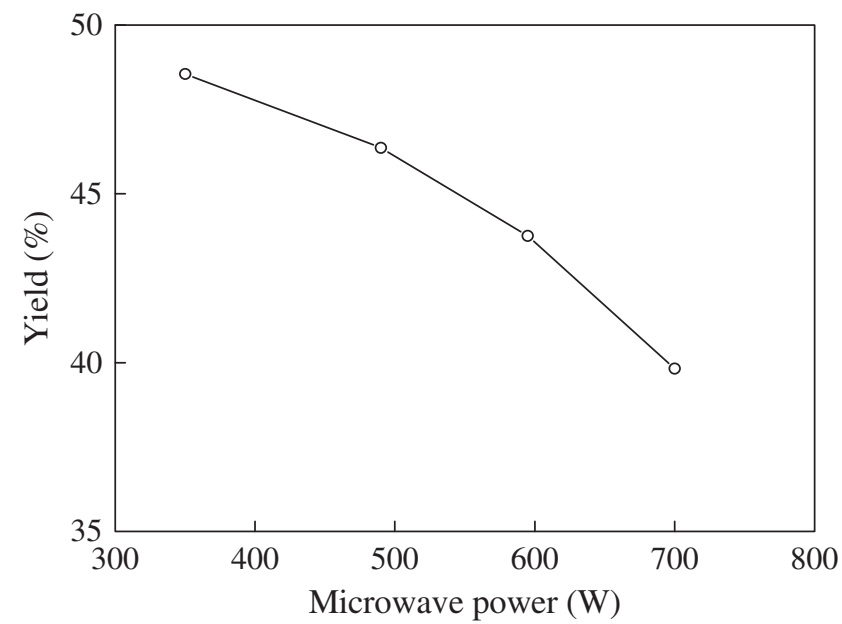

Figure 4: Effect of microwave power on the activation yield of activated carbon.

\section{Effect of microwave heating time}

\section{Pore structure analysis}

Figure 5 shows the nitrogen adsorption isotherms of the activated carbon prepared with different microwave heating times. Table 2 shows the details of pore structure while Figure 6 shows pore size distribution of activated carbon. The condition of experiment is microwave power of $700 \mathrm{~W}$.

It can be seen from Figure 5 that the type of isotherms are close to type I isotherms as referred to IUPAC classification. When relative pressure reaches 0.1 , adsorption is about $60 \%$ of saturation adsorption. With the relative pressure continuing to increase, adsorption increases

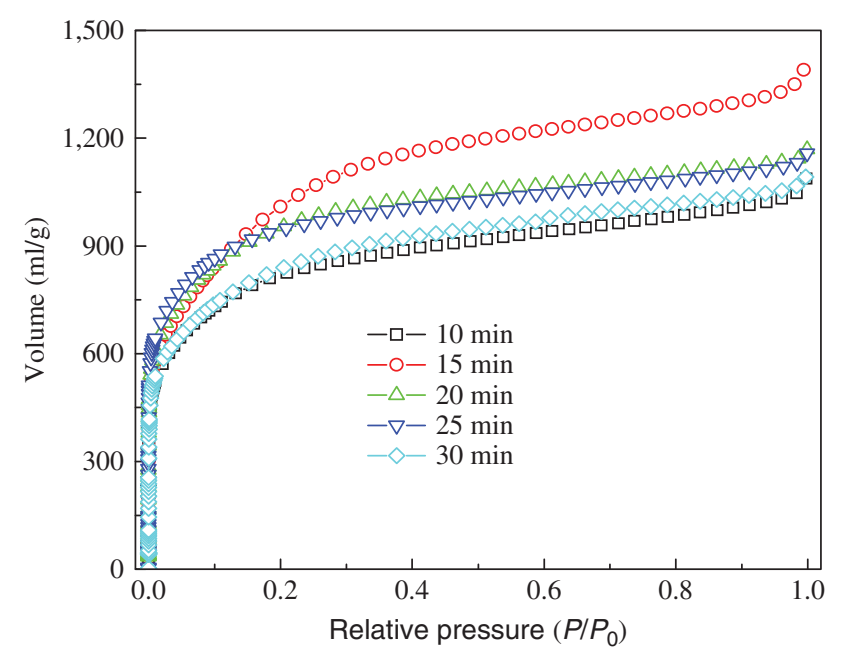

Figure 5: Nitrogen adsorption isotherm of the activated carbon with different microwave heating times. 
Table 2: Details of pore structure of activated carbon with different microwave heating times.

\begin{tabular}{lrrrrrrr}
\hline Heating time $(\mathrm{min})$ & $\boldsymbol{S}_{\text {BET }}\left(\mathrm{m}^{2} / \mathrm{g}\right)$ & $\boldsymbol{V}_{\text {tot }}(\mathrm{ml} / \mathrm{g})$ & $\boldsymbol{D}(\mathrm{nm})$ & $\boldsymbol{V}_{\text {mic }}(\mathrm{ml} / \mathrm{g})$ & $\boldsymbol{S}_{\text {mic }}\left(\mathrm{m}^{2} / \mathrm{g}\right)$ & $\boldsymbol{V}_{\text {mes }}(\mathrm{ml} / \mathrm{g})$ & $\boldsymbol{S}_{\text {external }}\left(\mathrm{m}^{2} / \mathrm{g}\right)$ \\
\hline 10 & 2,569 & 1.687 & 2.627 & 1.079 & 2,050 & 0.608 & 518.5 \\
15 & 3,441 & 2.093 & 2.434 & 1.304 & 2,591 & 0.789 & 850.1 \\
20 & 3,003 & 1.813 & 2.415 & 1.331 & 2,543 & 0.482 & 459.3 \\
25 & 2,896 & 1.796 & 2.480 & 1.275 & 2,422 & 0.521 & 474.6 \\
30 & 2,659 & 1.694 & 2.548 & 1.041 & 2,032 & 0.653 & 626.8 \\
\hline
\end{tabular}

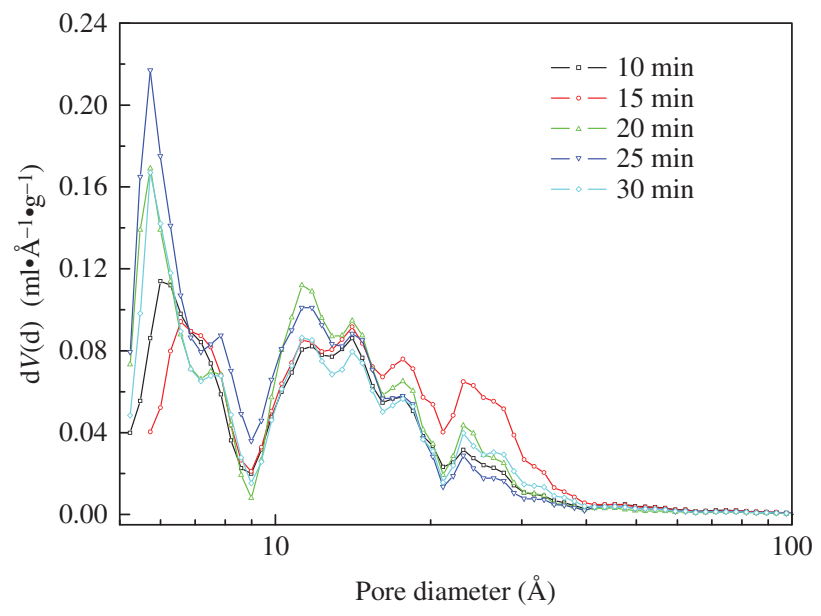

Figure 6: Pore size distribution chart for different microwave heating times.

slowly and the isotherm is seen as an upwardly convex shape. Adsorption ability increases quickly with relative pressure increase close to a $p / p_{0}$ of 1 .

It is shown in Table 2 that BET surface area, total pore volume, micropore surface area, external surface area, micropore volume and mesopore volume increase quickly when microwave heating time is less than $15 \mathrm{~min}$. When microwave heating time reaches around $15 \mathrm{~min}$, BET surface area, total pore volume and mesopore reach a maximum value. The micropore contents of activated carbon are beyond $60 \%$ at different heating times so that activated carbon could be seen in micropore style. Hence, microwave heating time has a significant effect to activated carbon.

Figure 6 indicates that pore size distribution is concentrated in micropore field according to IUPAC classification. The mesopore distribution around $20-40 \AA$ is abundant. Pore volume becomes very small when pore size is beyond $40 \AA$.

\section{FTIR analysis}

Figure 7 shows the FTIR analysis of the activated carbon at different microwave heating times. It can be seen from
Figure 7 that FTIR spectra of activated carbon at different microwave heating times are similar, with the major peaks observed at 3,438, 1,630, 1,300-1,000 and 917-620 $\mathrm{cm}^{-1}$. The band at around $3,438 \mathrm{~cm}^{-1}$ can be assigned to the $-\mathrm{OH}$ stretching vibration mode of hydroxyl functional groups. The band at around $1,630 \mathrm{~cm}^{-1}$ can be assigned to $C=\mathrm{C}$ symmetrical stretching of pyrone and $C=0$ of carboxylic groups and the band at $1,200-1,000 \mathrm{~cm}^{-1}$ is assigned to the $\mathrm{C}-\mathrm{O}$ stretching vibration mode. The adsorption peaks within $917 \mathrm{~cm}^{-1}$ are weak. When the microwave heating time becomes shorter, the $\mathrm{C}-\mathrm{H}$ outer surface rocking vibration absorption is weaker.

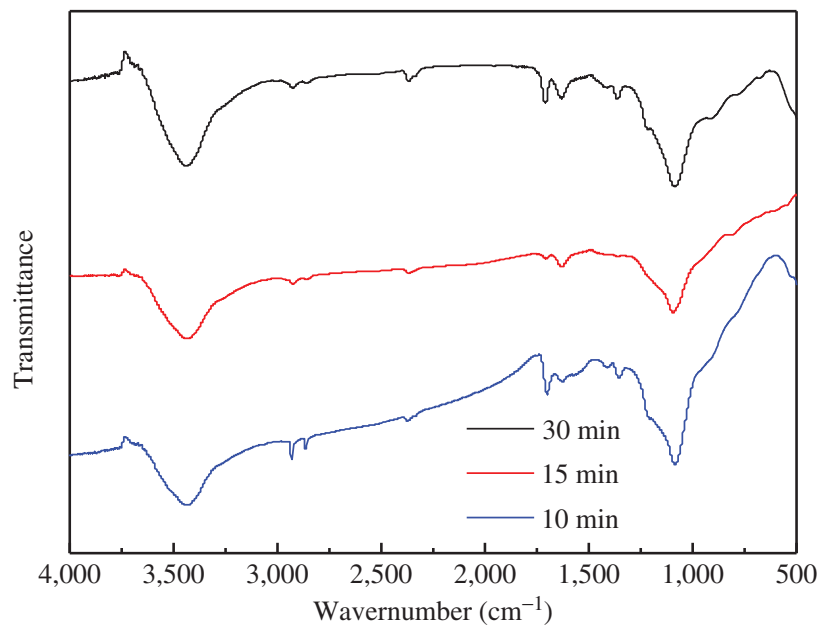

Figure 7: FTIR spectra of activated carbon at different microwave heating times from waste bamboo.

\section{Activation yield analysis}

Figure 8 illustrates the effects of microwave heating time on the activation yield of activated carbon. As can be seen from Figure 8, activation yield of activated carbon decreases when microwave heating time increases. When the heating time becomes longer and longer, the activating effect is better and ignition loss degree of activated carbon is larger. Similar yields have been reported in 


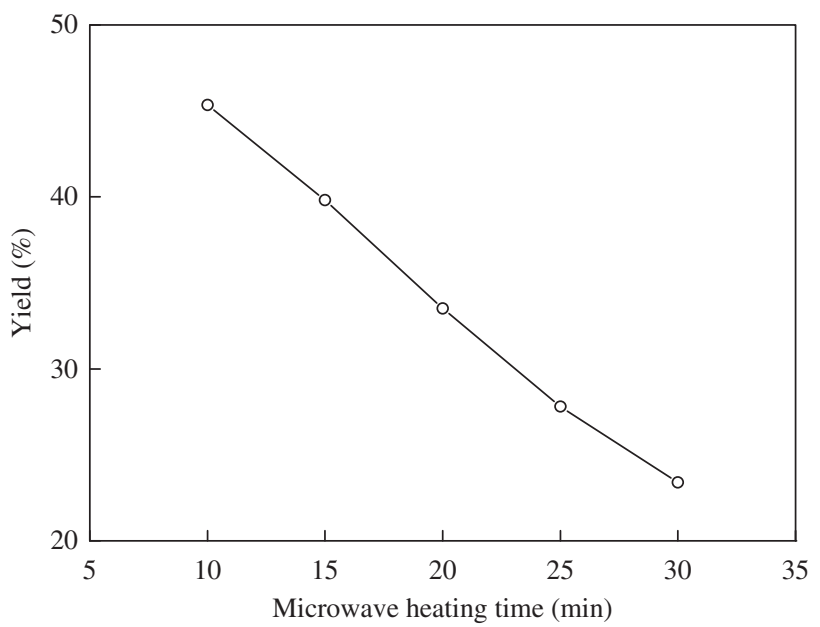

Figure 8: Effect of microwave heating time on the activation yield of activated carbon.

literatures for other precursors, namely for crofton $(18.03 \%)$ [33].

\section{Research analysis}

Table 3 compares the surface area of various activated carbons prepared using $\mathrm{KOH}$ activating agent utilizing different precursors as reported in the literature. Although it is a small sample, from the large number of activated carbon reported in the literature, the high surface area of the activated carbon prepared in the present work as compared with the literature is clearly evident. A surface as high as $3,441 \mathrm{~m}^{2} / \mathrm{g}$ is very rarely reported in the literature, which could form the basis for additional work on exploring the combination of bamboo precursor with the $\mathrm{KOH}$ activating agent.

\section{SEM analysis}

Figure 9 shows the SEM analysis of activated carbon. As can be seen from Figure 9, it is a picture of activated

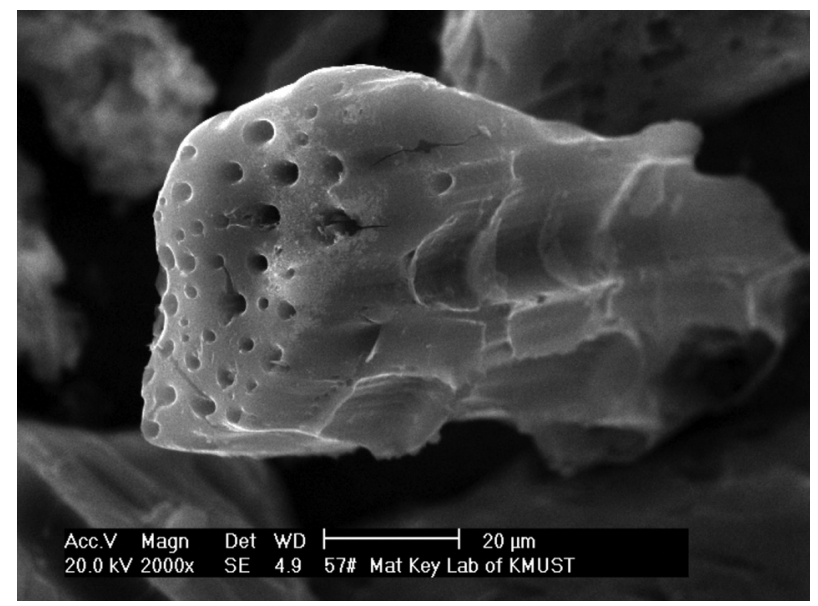

Figure 9: SEM image of activated carbon.

carbon particle. The activated carbon attests a significant development of pore structure and the impurities on the surface of the activated carbon are less.

\section{Conclusions}

Effect of microwave heating time and microwave power to bamboo-based activated carbon was discussed. The optimal conditions were microwave heating time of 15 $\mathrm{min}$ and microwave power of $700 \mathrm{~W}$ with $\mathrm{KOH} / \mathrm{C}$ ratio of 4. At the optimal conditions, surface area and pore volume were estimated to be $3,441 \mathrm{~m}^{2} / \mathrm{g}$ and $2.093 \mathrm{ml} / \mathrm{g}$, respectively. The characterization of FTIR illustrated that surface of activated carbon had abundant functional groups. The yield of activated carbon decreased with microwave heating time and microwave power increased. SEM analysis shows that activated carbon has a clear pore structure.

Acknowledgments: The authors express their gratitude to the Specialized Research Fund for the Doctoral Program

Table 3: Comparison surface area of activated carbon using $\mathrm{KOH}$ activating agent.

\begin{tabular}{lllrl}
\hline Activating agent & Precursors & Heating method & $\boldsymbol{S}_{\text {BET }}\left(\mathbf{m}^{2} / \mathbf{g}\right)$ & References \\
\hline $\mathrm{KOH}$ & Bamboo & Microwave heating & 3,441 & Present study \\
& Coconut husks & Microwave heating & 1,356 & {$[14]$} \\
& Isotropic petroleum pitch & Conventional heating & 2,992 & {$[15]$} \\
& Rambutan & Microwave heating & 971.54 & {$[16]$} \\
& Anthracite & Microwave heating & 3,451 & {$[17]$} \\
& Rice husk & Conventional heating & 2,696 & {$[18]$} \\
\hline
\end{tabular}


of Higher Education of China (No. 20115314120014), the Yunnan Provincial Science and Technology Innovation Talents scheme-Technological Leading Talent (No. 2013HA002) and the Kunming University of Science and Technology Personnel Training Fund (No. KKSY201252077) for financial support.

\section{References}

1. Gueye M, Richardson Y, Kafack FT, Blin J. High efficiency activated carbons from African biomass residues for the removal of chromium (VI) from wastewater. J Environ Chem Eng 2014;2:273-81.

2. Gratuito MKB, Panyathanmaporn T, Chumnanklang R-A, Sirinuntawittaya N, Dutta A. Production of activated carbon from coconut shell: Optimization using response surface methodology. Bioresour Technol 2008;99:4887-95.

3. Wang ZH, Chen Y, Zhou C, Whiddon R, Zhang YW, Zhou JH, et al. Decomposition of hydrogen iodide via wood-based activated carbon catalysts for hydrogen production. Int J Hydrogen Energy 2011;36:216-23.

4. Alicia Martínez de Yuso BR, Teresa Izquierdo M. Influence of activation atmosphere used in the chemical activation of almond shell on the characteristics and adsorption performance of activated carbons. Fuel Process Technol 2014;119:74-80.

5. ElShafei GMS, El Sherbiny IMA, Darwish AS, Philip CA. Silkworms' feces-based activated carbons as cheap adsorbents for removal of cadmium and methylene blue from aqueous solutions. Chem Eng Res Des 2014;92: 461-70.

6. Li HT, Zhang QS, Huang DS, Deeks AJ. Compressive performance of laminated bamboo. Composites B 2013;54:319-28.

7. Wang R, Amano Y, Machida M. Surface properties and water vapor adsorption-desorption characteristics of bamboo-based activated carbon. J Anal Appl Pyrol 2013;104:667-74.

8. Wang LG. Application of activated carbon derived from 'waste' bamboo culms for the adsorption of azo disperse dye: Kinetic, equilibrium and thermodynamic studies. J Environ Manage 2012;102:79-87.

9. Ahmad AA, Hameed BH. Fixed-bed adsorption of reactive azo dye onto granular activated carbon prepared from waste. J Hazard Mater 2010;175:298-303.

10. Asada T, Ohkubo T, Kawata K, Oikawa K. Ammonia adsorption on bamboo charcoal with acid treatment. J Health Sci 2006;52:585-9.

11. Danisha M, Hashim R, Mohamad Ibrahim MN, Sulaiman 0 . Effect of acidic activating agents on surface area and surface functional groups of activated carbons produced from Acacia mangium wood. Anal J Appl Pyrol 2013;104:418-25.

12. Auta M, Hammed BH. Preparation of waste tea activated carbon using potassium acetate as an activating agent for adsorption of Acid Blue 25 dye. Chem Eng J 2011;171:502-9.

13. Hao WM, Björkman E, Lilliestråle M, Hedi N. Activated carbons prepared from hydrothermally carbonized waste biomass used as adsorbents for $\mathrm{CO}_{2}$. J Anal Appl Pyrol 2014;105:166-76.

14. Suzuki RM, Andrade AD, Sousa JC, Rollember MC. Preparation and characterization of activated carbon from rice bran. Bioresour Technol 2007;98:1985-91.
15. Foo KY, Hameed BH. Coconut husk derived activated carbon via microwave induced activation: Effects of activation agents, preparation parameters and adsorption performance. Chem Eng J 2012;184:57-65.

16. Njoku VO, Koo KY, Hameed BH. Preparation of activated carbons from rambutan (Nephelium lappaceum) peel by microwaveinduced $\mathrm{KOH}$ activation for acid yellow 17 dye adsorption. Chem Eng J 2014;250:198-204.

17. Zhao W, Fierro V, Fernández-Huerta N, Izquierdo MT, Celzard A. Impact of synthesis conditions of $\mathrm{KOH}$ activated carbons on their hydrogen storage capacities. Int J Hydrogen Energy 2012;37:14278-84.

18. Muniandy L, Adam F, Mohamed AR, Eng-Poh NG. The synthesis and characterization of high purity mixed microporous/ mesoporous activated carbon from rice husk using chemical activation with $\mathrm{NaOH}$ and $\mathrm{KOH}$. Micropor Mesopor Mat 2014;197:316-23.

19. Vilaplana-Ortego E, Lillo-Ródenas MA, Alcañiz-Monge J, Cazorla-Amorós D, Linares-Solano A. Isotropic petroleum pitch as a carbon precursor for the preparation of activated carbons by KOH activation. Carbon 2009;47: 2112-43.

20. Oliveira LCA, Pereira E, Guimaraes IR, Vallone A, Pereira M, Mesquita JP, et al. Preparation of activated carbons from coffee husks utilizing $\mathrm{FeCl}_{3}$ and $\mathrm{ZnCl}_{2}$ as activating agents. J Hazard Mater 2009;165:87-94.

21. Mestre AS, Bexiga AS, Proença M, Andrade M, Pinto ML, Matos I, et al. Activated carbons from sisal waste by chemical activation with $\mathrm{K}_{2} \mathrm{CO}_{3}$ : Kinetics of paracetamol and ibuprofen removal from aqueous solution. Bioresour Technol 2011;102:8253-60.

22. Xiao H, Peng H, Deng SH, Yang XY, Zhang YZ, Li YW. Preparation of activated carbon from edible fungi residue by microwave assisted $\mathrm{K} 2 \mathrm{CO} 3$ activation-Application in reactive black 5 adsorption from aqueous solution. Bioresour Technol 2012;111:127-33.

23. Baccar R, Bouzid J, Feki M, Montiel A. Preparation of activated carbon from Tunisian olive-waste cakes and its application for adsorption of heavy metal ions. J Hazard Mater 2009;162:1522-9.

24. Duan XH, Srinivasakannan C, Qu WW, Wang X, Peng JH, Zhang LB. Regeneration of microwave assisted spent activated carbon: Process optimization, adsorption isotherms and kinetics. Chem Eng Process 2012;53:53-62.

25. Yang KB, Peng JP, Srinivasakannan C, Zhang LB, Xia HY, Duan $\mathrm{XH}$. Preparation of high surface area activated carbon from coconut shells using microwave heating. Bioresour Technol 2010;101:6163-9.

26. Ferrera-Lorenzo N, Fuente E, Suárez-Ruiz I, Ruiz B. KOH activated carbon from conventional and microwave heating system of a macroalgae waste from the Agar-Agar industry. Fuel Process Technol 2014;121:25-31.

27. Yürüm A, Özlem Kocabaş-Ataklı Z, Sezen M, Semiat R, Yürüm Y. Fast deposition of porous iron oxide on activated carbon by microwave heating and arsenic (V) removal from water. Chem Eng J 2014;242:321-32.

28. Ahmed MJ, Samar K. Theydan.Optimization of microwave preparation conditions for activated carbon from Albizia lebbeck seed pods for methylene blue dye adsorption. J Anal Appl Pyrol 2014;105:199-208. 
29. Sun YY, Yue QY, Mao YP, Gao BY, Gao Y, Huang LH. Enhanced adsorption of chromium onto activated carbon bymicrowaveassisted $\mathrm{H}_{3} \mathrm{PO}_{4}$ mixed with $\mathrm{Fe} / \mathrm{Al} / \mathrm{Mn}$ activation. J Hazard Mater 2014;265:191-200.

30. Pezoti Jr O, Cazetta AL, Gomes RC, Barizão EO, Souza IPAF, Martins AC, et al. Synthesis of $\mathrm{ZnCl} 2$-activated carbon from macadamia nut endocarp (Macadamia integrifolia) by microwave-assisted pyrolysis: Optimization using RSM and methylene blue adsorption. J Anal Appl Pyrol 2014;105: 166-76.
31. IUPAC. Manual of symbols and terminology of colloid surface. London: Butterworths, 1982.

32. Gomez-Serrano V, Cuerda-Correa EM, Fernandez-Gonzalez CM, Alexandre-Franco MF, Macias-Garcia A. Preparation of activated carbons from chestnut wood by phosphoric acid-chemical activation. Study of microporosity and fractal dimension. Mater Lett 2005;59:846-53.

33. Zheng ZQ, Xia HY, Srinivasakannan C, Peng JH, Zhang LB. Utilization of Crofton weed for preparation of activated carbon by microwave induced $\mathrm{CO}_{2}$ activation. Chem Eng Process 2014;82:1-8. 